\title{
A Survey on SPIN Protocol in Wireless Sensor Network
}

\author{
Mohammed Salamath ${ }^{1}$, Sunitha $\cdot \mathbf{R}^{2}$ \\ ${ }^{1}$ PG Student, Dept. of CSE, MCE, Hassan, India \\ ${ }^{2}$ Assistant Professor, Dept. of CSE.MCE, Hassan, India
}

\begin{abstract}
Wireless sensor networks consisting of sensor nodes, which are all contribute themselves in effective data communication. By sensing the data and processing the data that's why Wireless Sensor Networks are adapted in the field of health monitoring, rescue operations and disaster management. One of the most important task in wireless sensor network is efficient data transmission, for this purpose many energy efficient routing protocols are proposed. Among those here in this paper one of the data centric routing protocol that is SPIN routing protocol is surveyed with respect to their family and at last proposed a secure - SPIN routing protocol which elaborates about some improvements to be made to achieve high scalability and high network lifetime.
\end{abstract}

Keywords: SPIN, Secure - SPIN, wireless sensor network, data centric routing protocol

\section{Introduction}

Wireless sensor networks comprises of small sensor nodes[1], which measures environments parameters such as Humidity, vibration, sound and few other parameters. Numerous civil and military applications can be leveraged by network sensors. SPIN is a negotiation based protocol which includes negotiation and resource adaptation. In negotiation each node negotiate with other nodes before they transmit the data and resource adaptation means each node have resource manager that keeps track of resource usage.

In this paper survey is done on current routing protocols of SPIN and proposed a new routing protocol for SPIN called Secure-SPIN. The paper is organized as follows. First I have identified the wireless sensor network routing protocols then presented the current routing protocols after that I have extended these routing protocols into high scalable and high network lifetime protocol.

\section{Working of Wireless Sensor Network}

In WSN each node senses the data from environment and processes it and finally sends it to the base station. These nodes are pivot elements of wireless network, then from the base station user can get the data. The figure 1 shows the components of sensor node. Basically it includes processing, sensing, mobilizer, transmission, position finding system and power units. And that figure itself shows the architecture of the wireless sensor network also.

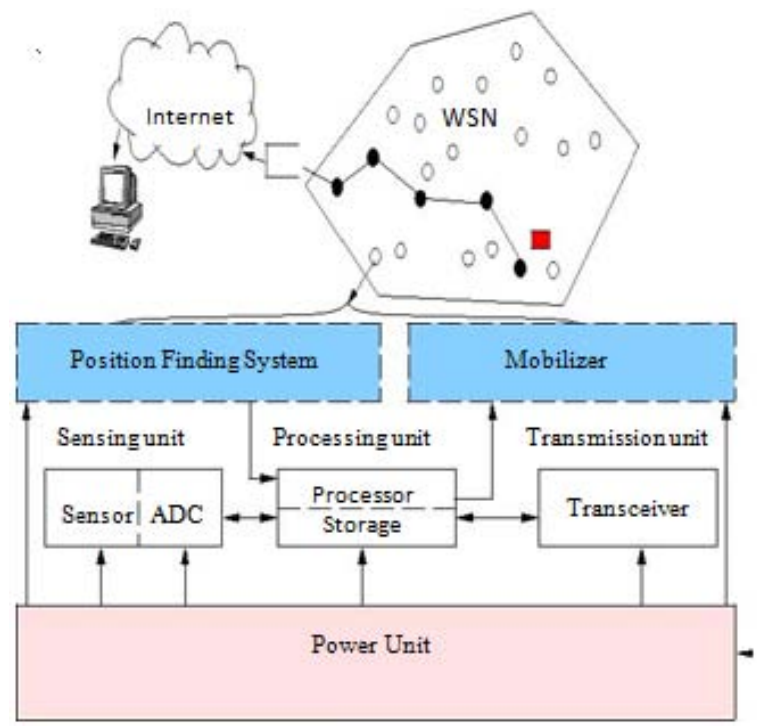

Figure 1: The components of a sensor node

\section{Wireless Sensor Network Routing Protocols}

In wireless sensor network many routing protocols are introduced [5] to overcome collision, accelerate transmission and to save energy of a node. As shown below, in this paper only SPIN routing protocol family are discussed.

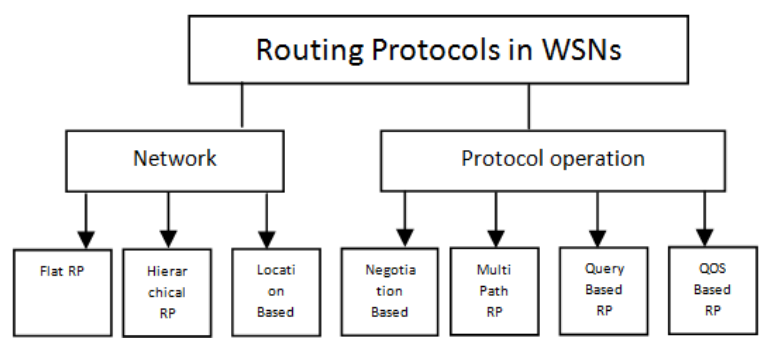

Figure 2: Classification of routing protocols in WSNs

A. Sensor protocol for information via negotiation (SPIN)

SPIN is one of the routing protocols in wireless sensor network [1], [2], [3], which spreads all of the information 


\section{International Journal of Science and Research (IJSR) \\ ISSN (Online): 2319-7064}

Index Copernicus Value (2013): 6.14 | Impact Factor (2014): 5.611

at each sensor node to every other node in the network. Here there is one assumption that all nodes in a network are base stations. This allows a user to request any node and get required information quickly. SPIN is a negotiations and resource adaptive protocol that is used to solve the problem flooding which causes implosion problem. This implosion problem makes wastage of resources such as energy and bandwidth.

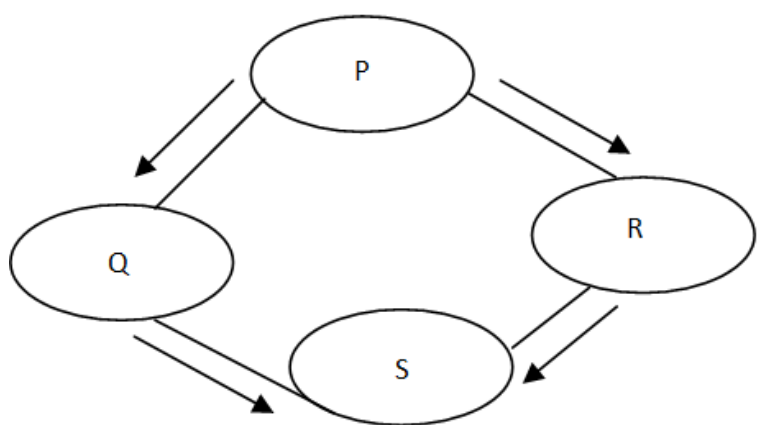

Figure 3: The implosion problem (Overlapping of Data)

\section{B. Working of SPIN}

It transmits the information by the procedure called negotiating [6]. For this purpose SPIN uses metadata (Partial description about the data) first a node broadcast the metadata to other nodes, if a particular node wishes to receive actual data then only it sends that data by using following three messages.

ADV: This message used by a node to advertise metadata among neighbor.

REQ: This message used to request a data.

DATA: This message used to send actual data message.

This protocol starts when a node sense the new data it broadcast the ADV message containing (Partial description about the data) called metadata. If any neighbor node interested in the data it sends a REQ message and get back the data with the help of DATA message to this neighbor node. Then this neighbor node repeats this procedure until all the nodes in the wireless sensor network get a copy of the data.

If the topology of the network changes it can be localized because each node need to know only its single hop neighbors. Data advertisement in SPIN cannot assure about delivery of the data. The SPIN protocol family consisting of seven protocols, SPIN -PP, SPIN-BC, SPINRL, SPIN-EC, Modified SPIN and Cluster-SPIN, SecureSPIN.

\section{SPIN-PP}

SPIN-PP is design for a point to point communication network. It uses three step hand shake protocol and reduces energy consumption of a node in wireless sensor network.
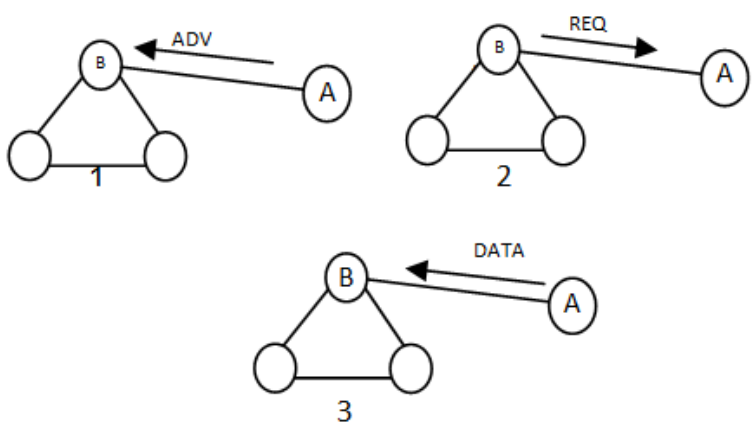

Figure 4: SPIN-PP Protocol- Node A send ADV message to node $\mathrm{B}(1)$. Node $\mathrm{B}$ provide response to node $\mathrm{A}$ with REQ message(2) after receiving the data request node $A$ sends DATA message to node $\mathrm{B}(3)$

One node can communicate with other node without interfering the other nodes in the network. SPIN-PP is applicable only in preconfigured network with less cost in the perspective of finding neighbor node. So they can spent little energy in computation because each node has to know only one hop neighbor node in the network.

\section{SPIN-EC}

It is a energy conservation [11] to the SPIN-PP protocol. Hence a node can decide whether he may participate or not in communication based on the energy threshold level of it, if a node have high threshold energy then it may involve for sending and receiving the messages otherwise can't. But in this protocol does not prevent a node from receiving, this accounts energy expenditure on reception.

\section{E. SPIN-BC}

It is designed for broadcast networks [11]. Here it shares a only one channel for communications. If a node sends a data on broadcast channel the data is received by all other nodes which are in a range of the sending node.

If a particular node sends the ADV message to its neighbor in its range, a neighbor nodes after receiving ADV message they won't send REQ message immediately, they wait for some time and analyses the network whether any other node sending the REQ or note, if other node sent the REQ then it won't send and that other node receives a data message upon its REQ message. So in this way remaining nodes can eliminates them in receiving same message as well as energy of a nodes can also be saved.

\section{F. SPIN-RL}

It extends the capability of SPIN-BC to increase its reliability. In this protocol it periodically broadcast the ADV and REQ messages and each node keep track of it. If node requested a data from other node and data messages are not arrived to that node in intime, then REQ message can be resend. So reliability can be improved by readvertising metadata periodically.

G. M-SPIN (Modified SPIN) 


\section{International Journal of Science and Research (IJSR) \\ ISSN (Online): 2319-7064 \\ Index Copernicus Value (2013): 6.14 | Impact Factor (2014): 5.611}

The energy can be saved [8], if we control the number of transmission and reception of messages in a network. In M-SPIN distance discovery phase is introduced which is not included in above protocols. According to this phase it finds a shortest path among nodes to base station for communication. In distance discovery phase number of hop determines a shortest path, based upon this negotiation phase is illustrated. It is a selective transmission. But it is also having some limitations, that is every time same shortest path was chosen for communication these leads to energy lose in the shortest path nodes.

\section{The disadvantages of SPIN family}

- The dissemination of data in the network through SPIN protocol takes long time.

- A node with much more computation consumes more energy.

- Few sensor nodes may be used several times and those nodes may lose energy early then other nodes in the network.

- Energy will be reduced without transmission of data by nodes sitting idle.

\section{H. C-SPIN (Cluster version of SPIN)}

This method [4] restricts the usage of same shortest path. This is a cluster version of SPIN protocol. It also uses three stages that is ADV, REQ and DATA stage to do communication including clustering algorithms are used for efficient data transmission. This method guarantees the data delivery. Here network is divided into clusters and each cluster get communicated by their respective cluster head's and each time cluster head is also rotated randomly to save energy. Here at any point of time cluster head become dead. So that is the main limitation of the protocol.

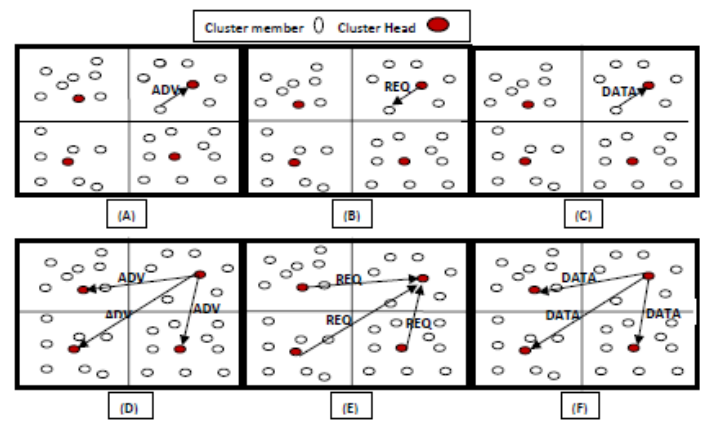

Figure 5: Network is partitioned into clusters initially

1. A cluster member have new sense data, it sends ADV message to $\mathrm{CH}$.

2. $\quad \mathrm{CH}$ replies REQ message.

3. Cluster member (CM) sends data to $\mathrm{CH}$ by DATA message.

4. $\quad \mathrm{CH}$ sends $\mathrm{ADV}$ message to other neighbor $\mathrm{CH}$ 's in the network.

5. Neighbor $\mathrm{CH}$ provide response with REQ message.

6. Then initial $\mathrm{CH}$ sends data packet with the help of DATA message.

\section{S-SPIN (Secure SPIN)}

Here secure SPIN protocol is proposed which have all the features of above but in this scheme to increase the life time of the network and to achieve scalability, a dead nodes including cluster heads are all dynamically replaced by using recovery and replace algorithm.

\section{Advantages of cluster based SPIN and secure SPIN}

- Energy expenditure during transmission among the nodes is less to reserve their energy.

- Each and every node will become a cluster head exactly once every rounds.

- Guarantees the data delivery from source node to base station.

- Reduces the intercommunications distance.

\section{Simulation}

To do the performance test of SPIN protocol family NS2.34 is used.NS2 is an event simulator which provides efficient support for simulation of routing protocols over wireless networks.

\section{a. Performance matrices}

Performance matrices to evaluate the SPIN routing family protocols are as follows:-

- Packet delivery ratio:- Ratio of packets that are successfully delivered to a destination compared to the number of packets that have been sent out by the sender.

- End to End delay:- Average time taken by a data packet to arrive in a destination, lower value of end to end delay gives higher performance.

- Energy spent:- Total amount of energy spent during transmission and reception of messages in node.

- Still so many performance matrices are there to evaluate it. Here only packet data ratio and energy consumption are considered.

\section{b. Result Analysis}

This section discusses the results which are obtained after simulating the wireless sensor networks using SPIN family protocols.

The simulation factors are listed in the below Table 1 .

Table 1: Simulation Factors

\begin{tabular}{|c|c|}
\hline Factors Name & Scenario \\
\hline Channel Type & Wireless channel \\
\hline Radio Propagation model & TwoRayGround \\
\hline Network Type & Static and mobile \\
\hline Number of Node & 50 \\
\hline Simulation Time & 150 Sec \\
\hline Initial Energy & $100 \mathrm{~J}$ \\
\hline
\end{tabular}




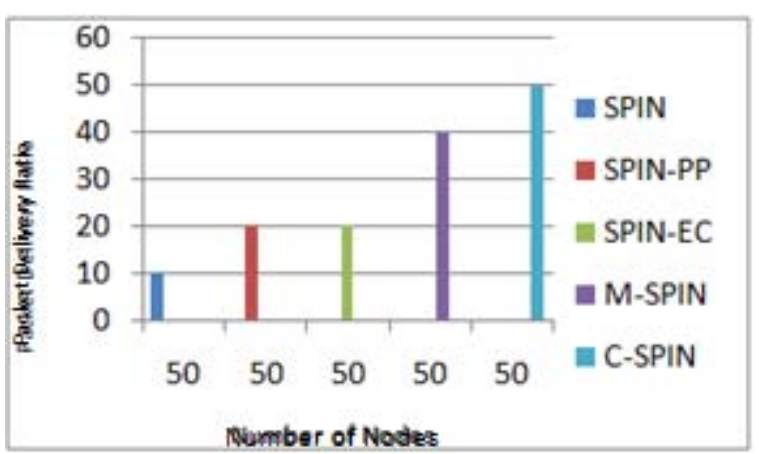

Figure 6: Packet data ratio with number of nodes

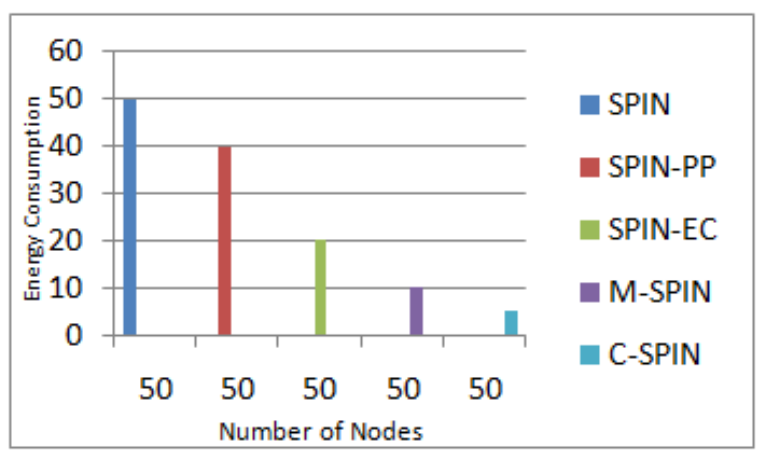

Figure 7: Energy consumption with number of nodes

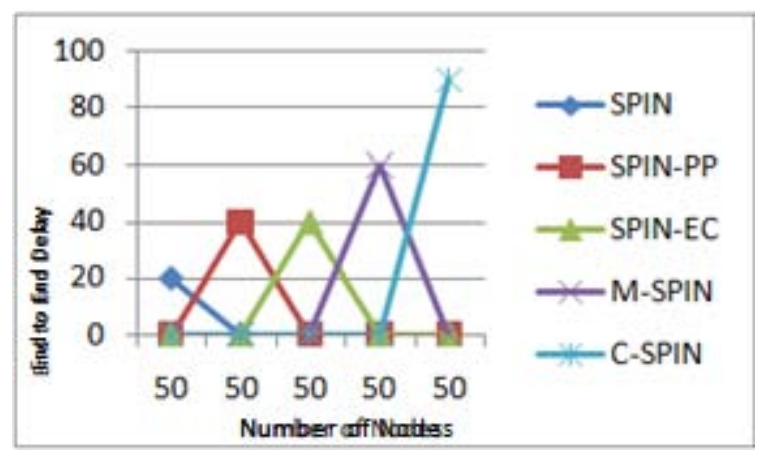

Figure 8: Packet delivery ratio of SPIN protocol family

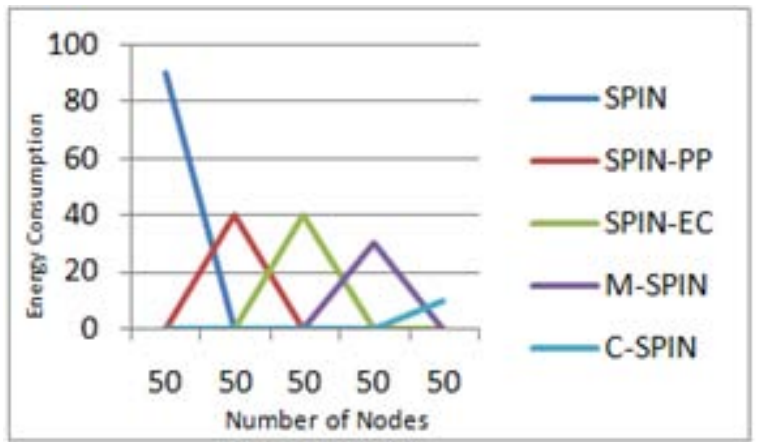

Figure 9: Energy consumption of SPIN protocol family

- SPIN-PP reduces energy by $40 \%$ and no redundant data.

- SPIN-EC distributes $20 \%$ additional data compared to SPIN-PP.

- SPIN-BC reduces energy by 50\% and moderate packet delivery ratio.

- SPIN-RL 99\% packet delivery ratio and consumes more energy.

- C-SPIN consumes less energy and good packet delivery ratio.

\section{Simulation Summary}

Simulation results of above SPIN protocol family has been tested with network size 50 nodes and packet size 100 bytes. Result shows that SPIN, SPIN-PP, SPIN-EC, and SPIN-BC gives worst performance where M-SPIN and CSPIN gives Best performance.

\section{Conclusion}

In this paper SPIN protocol family was surveyed and proposed a new protocol called cluster-secure SPIN, which not only guarantees a data delivery it also increases life time of the network by recovering and replacing a nodes dynamically.

\section{References}

[1] I.Akyildiz, W. Su, Y. Sankarasubramaniam, and E. Cayirci, "A survey on sensor networks," IEEE Communications Magazine, vol. 40, no. 8,pp. 102114, August 2002.

[2] Malik Tubaishat and Sanjay Madria, "Sensor Networks: An Overview," IEEE Potential, pp. 20- 23, 2003.

[3] Holger Karl, and Andreas Willig, "Protocol and Architecture for Wireless Sensor Networks," Wiley Publication, 2005.

[4] Ashutosh Tripathi, Narendra Yadav, Reena Dadhich "Optimization of Clustering in SPIN-C and LEACH for Data Centric Wireless Sensor Networks," Proceedings of Fourth International Conference on Soft Computing for Problem Solving, pp 197-205, Springer,2015

[5] K. C. Rahman, "A Survey on Sensor Network", JCIT, ISSN 2078-5828 (PRINT), ISSN 2218-5224 (ONLINE), vol. 01, is. 01, Manuscript Code: 100715, (2010).

[6] Geetu and Sonia juneja, "a study on a routing protocol SPIN in WSN”,2012 vol 5,no 2, pp 345-352,IJITM.

[7] Martin Enzinger, "Energy-efficient communication in Wireless Sensor Networks", Proceedings of the Seminar Sensor Nodes - Operation,

[8] Zeenat Rehena, Sarbani Roy, Nandini Mukherjee, "A Modified SPIN for Wireless Sensor Networks", 9781-42448953-4/11 2011 IEEE.

[9] Kazi Chandrima Rahman, "A Survey on Sensor Network”,JCIT, ISSN 2 078-5828 (PRINT), ISSN 2 21 8-52 24(ONLINE), 1(1), manuscript code: 100715,2010

[10] S.K. Singh, M.P. Singh, and D.K. Singh, "A survey of Energy-Efficient Hierarchical Cluster-based Routing in Wireless Sensor Networks", International Journal of Advanced Networking and Application (IJANA), Sept.-Oct. 2010, vol. 02, issue 02, pp. 570-580.

[11]Kulik, Joanna, Wendi Heinzelman, and Hari Balakrishnan. "Negotiation-based protocols for disseminating information in wireless sensor networks." Wireless networks 8.2/3 (2002): 169-185 